\title{
Evaluación Dasométrica De Una Plantación De Pinus Radiata D. Don En EI Sector San Andrés, Guano, Ecuador
}

\author{
Miguel Angel Guallpa Calva (Ing. For. Mgs.) \\ Sonia Carmita Rosero Haro (Ing. Agr. M.Sc.) \\ Docentes investigadores, Facultad de Recursos Naturales/ \\ Escuela Superior Politécnica de Chimborazo/Ecuador
}

Doi: 10.19044/esj.2018.v14n15p78 URL:http://dx.doi.org/10.19044/esj.2018.v14n15p78

\begin{abstract}
There are scarce information concerning the growth and productivity of the trees of the plantation of Pinus radiata D. Don. This is usually found in the forest estate of the Haro-Maderas factory in a condition of soil with sandy loam texture, deep and moderately fast drainage, $\mathrm{pH} 6.7$, fertility between low and high with the specific application of a pruning activity at the age of 5 years, and gradual monitoring every year until 8 years of age. This is considered as part of its management which constitutes a benchmark in the Tahuala sector. For this reason, this study aims to evaluate its forest mass. Circular plots of $8.92 \mathrm{~m}$ radius were installed at an intensity of $4.33 \% \mathrm{ha}^{-1}$. This, however, is done through a systematic aligned sampling for the collection of dasometric information. The application of statistical estimators showed an altitude of 3145 masl. It reached an average total height of 12.76 $\mathrm{m}$, a DAP of $15.27 \mathrm{~cm}$, the total average tree- 1 estimated volume of $0.16 \mathrm{~m}^{3}$, and its projected value of $303.56 \mathrm{~m}^{3} \mathrm{ha}^{-1}$. The relationship between the DAPage variables and the average-age total height was high and significant. It has a correlation coefficient equal to 0.99 , whose estimation was better with the linear type equation. It was suggested that thinning should be carried out with an intensity of $28.77 \%$. In regards to the remaining trees, it will be very necessary to apply the silvicultural treatment of pruning.
\end{abstract}

Keywords: Dasometric, forest plantation, yield, Radiata pine, thinning index

\section{Resumen}

Existe escasa información sobre el crecimiento y productividad de los árboles de la plantación de Pinus radiata D. Don en el predio forestal de la Fábrica Haro-Maderas en una condición de suelo con textura franco arenosa, profundo, drenaje moderadamente rápido, $\mathrm{pH}$ 6.7, fertilidad entre baja y alta 
con la aplicación específica de una actividad de poda a la edad de 5 años y monitoreo paulatino cada año hasta los 8 años de edad como parte de su manejo orientado a constituir un referente del sector Tahuala; por esta razón, se decidió evaluar su masa forestal. Se instalaron parcelas circulares de 8.92 $\mathrm{m}$ de radio a una intensidad de $4.33 \% \mathrm{ha}^{-1}$. Mediante el muestreo sistemático alineado para el levantamiento de información dasométrica. La aplicación de estimadores estadísticos, determinó que a una altitud de $3145 \mathrm{msnm}$ alcanzó una altura total promedio de $12.76 \mathrm{~m}$, un DAP de $15.27 \mathrm{~cm}$, el volumen total promedio árbol ${ }^{-1}$ estimado de $0.16 \mathrm{~m}^{3} \mathrm{y}$ su valor proyectado $303.56 \mathrm{~m}^{3} \mathrm{ha}^{-1}$. La relación entre las variables DAP-edad y altura total promedio-edad fue alta y significativa, con coeficientes de correlación igual a 0,99 cuya estimación resulto mejor con la ecuación de tipo lineal. Se sugiere ejecutar un raleo con una intensidad del $28.77 \%$, luego al remanente de árboles será necesario aplicar el tratamiento silvicultural de poda.

Palabras-clave: Dasometría, plantación forestal, rendimiento, Pinus radiata, índice de raleo

\section{Introducción}

En Ecuador el 34,7\% de la superficie nacional está cubierta por bosques, el $98.5 \%$ son bosques naturales, las plantaciones no superan el 1,5 $\%$ restante del patrimonio forestal (Grijalva et al., 2012). Durante el período 2007-2010, la madera autorizada para aprovechamiento con el 58,5\% que correspondió a plantaciones forestales (Palacios \& Quiroz, 2012).

La especie Pinus radiata D. Don es una de las especies exóticas que mayormente predomina en climas templados y suelos diversos (Carle et al., 2009). Después del eucalipto es la segunda especie plantada en la serranía ecuatoriana de alta capacidad productiva que prospera en suelos de textura media con pH desde ácido a neutro y con buen drenaje. Entre 1.800 a 3.500 msnm, con una precipitación de 800 a $1.300 \mathrm{~mm}$ y una temperatura de 11 a $17^{\circ} \mathrm{C}$ (Ecuador Forestal, 2013). En la región sierra, es priorizada para la reforestación con fines comerciales debido a su adaptabilidad, rápido crecimiento y rentabilidad (MAGAP, 2015, p.42).

Actualmente es muy utilizada para la elaboración de tableros aglomerados y de fibra, se lo puede establecer en cortinas rompevientos, cercas vivas, sistemas silvopastoriles o en plantaciones puras. Para el periodo 2008 - 2009, se autorizó aprovechar 388,72 miles de $\mathrm{m}^{3}$ provenientes de las provincias de Chimborazo, Cotopaxi y Bolívar. Ocupando el tercer lugar con el $13,24 \%$ del volumen total de madera autorizada y aprovechada del país (MAE, 2010, p.11). 
La aplicación de técnicas al establecimiento y su manejo con actividades de limpieza en corona, raleo y podas influyen en el incremento medio anual con valores de 5 a $20 \mathrm{~m}^{3} \mathrm{ha}^{-1} \mathrm{año}^{-1}$ (Ecuador Forestal, 2013).

Según esto, la información existente sobre la dinámica de evolución de las variables dasométricas en su fase juvenil, limitan la posibilidad de estimar su crecimiento y productividad de los árboles de pino a nivel de sitio en el sector de Tahuala y otras áreas potenciales de la parroquia de San Andrés.

En este contexto, el presente estudio, se tradujo en la búsqueda de cuantificar la expresión del sitio que logra el bosque plantado en respuesta a la influencia edafoclimática del área geográfica en que se desarrolla y la ausencia de labores silviculturales, motivó evaluar su crecimiento y productividad, para disponer de información técnica orientada a la estimación de la productividad forestal y su posterior manejo como rodal de producción. Este hecho se justifica por aspectos: tamaño de la propiedad, cuidados silviculturales escasos o inexistentes (Diaz Balteiro et al., 2008). Por ende es fundamental ejecutar la administración, el manejo del bosque, a fin de obtener una óptima y continua productividad del sitio, con las condiciones tecnológicas y socio económicas prevalecientes (Hernández, 2014).

A partir del muestreo de la plantación, se levantó la información considerando los parámetros dasométricos de la especie (Nájera \& Hernández, 2008). El objetivo del presente estudio fue evaluar el crecimiento a partir del diámetro a la altura del pecho y altura total promedio de Pinus radiata D. Don en función a la edad, su grado de correlación y la productividad mediante el área basal, volumen total proyectado al reportar valores superiores en relación a estimaciones de rendimiento en el sector de Mulaló a similar altitud y edad de la plantación. Adicionalmente, se calculó el índice de raleo como aporte prioritario en la aplicación de técnicas silvícolas, para mejorar su productividad, lo que influye en la rentabilidad final (Sharma et al., 2001).

\section{Materiales y Métodos}

La presente investigación se realizó en la plantación de $P$. radiata $\mathrm{D}$. Don de 8 años de edad, ubicada en el predio experimental de la Fábrica Haro Maderas, sector Tahuala, parroquia San Andrés, cantón Guano, provincia de Chimborazo-Ecuador, a $17 \mathrm{~km}$ de la ciudad capital. Geográficamente la masa forestal se inserta dentro de los puntos de referencia: UTM Zona 17S Datum WGS $84 X_{1}=763609 ; Y_{1}=9806880, X_{2}=764973 ; Y_{2}=9806791, X_{3}=$ $764973 ; \mathrm{Y}_{3}=9805248$ y $\mathrm{X}_{4}=763606 ; \mathrm{Y}_{4}=9805251$.

Se encuentra ubicada a una altitud que va desde $3137-3146 \mathrm{msnm}$. La precipitación anual es $560,1 \mathrm{~mm}$, siendo la temperatura media $13,3^{\circ} \mathrm{C}$ y una humedad relativa media de 85\%. Datos de la Estación Meteorológica de la ESPOCH; serie 1984-2014. La plantación tiene una superficie neta de 1,48 ha, 
y se categoriza dentro de la formación: Bosque Siempre verde Montano Alto de los Andes Orientales (Sierra et al., 1999).

El área de plantación presentó las siguientes características fisiográficas: Una altitud de $3146 \mathrm{msnm}$, siendo la pendiente menor al 5\%, con un nivel de no pedregoso a nivel interno (<5\%) y un drenaje moderadamente rápido $\left(6,30-12,70 \mathrm{~cm} \mathrm{hr}^{-1}\right)$.

Dentro de los parámetros edáficos tiene una profundidad mayor a 100 $\mathrm{cm}$, se aprecia condición textural franca arenosa con una estabilidad estructural baja en el suelo de la plantación. La densidad aparente de 1,4 g $\mathrm{cm}^{3-1}$ y una densidad real de $2,5 \mathrm{~g} \mathrm{~cm}^{3-1}$.

Al determinar las propiedades químicas a los 3 y 4 años de haber sido establecida la plantación el contenido de materia orgánica sus valores oscilan entre 2 a $0,6 \%$ ubicándose en un nivel bajo $(<3 \%)$. Se encontró que el potencial de hidrógeno $(\mathrm{pH})$ es ácido desde 6,7 a 6,$2 ;$ los contenidos nutricionales: el nitrógeno entre $10-4,8$ como nivel bajo $\left(<30.00 \mathrm{mg} \mathrm{L}^{-1}\right)$; el fósforo alto: 52,8 hasta 64,8 (> 30,00 $\mathrm{mg} \mathrm{L}^{-1}$ ); el potasio alto desde 1,34 hasta 1,49(>0,64 Meq100 $\left.\mathrm{g}^{-1}\right)$. Resultados reportados en el laboratorio de suelos de la Facultad de Recursos Naturales-ESPOCH. 2012.

\section{Prácticas empleadas en el establecimiento y manejo de la plantación}

El bosque plantado fue establecido con un sistema cuadrado de $2,15 \mathrm{x}$ 2,15 m con un nivel de prendimiento del 93,14\% en la primera evaluación (2009) disminuyendo hasta el $87,53 \%$ en la octava evaluación realizada en el año 2016.

No se realizó fertilización, coronamiento y otras actividades de manejo: raleos, solo existe un reporte de aplicación de poda a la edad de 5 años y monitoreo paulatino cada año hasta los 8 años de edad.

\section{Métodos}

El muestreo de las variables dasométricas del DAP y altura total tuvo lugar el mes de octubre de cada año desde el año 2009 hasta el año 2016. Datos utilizados para realizar el cálculo de crecimiento, correlación de las variables: diámetro a la altura del pecho, altura total en función del tiempo, y con los datos obtenidos en el año 2016 se determinó las variables de productividad y el cálculo del índice de raleo.

\section{Cobertura forestal}

Se instalaron tres parcelas circulares con un radio de 8,92 $\mathrm{m} \mathrm{y}$ separadas entre ellas a $50 \mathrm{~m}$, con el diseño de muestreo sistemático (FAO, 2011); considerando la topografía y exposición del rodal aplicando una intensidad de muestreo del $4,33 \% \mathrm{ha}^{-1}$. 
En las parcelas se contaron los árboles fustales $(\varnothing>$ a $8 \mathrm{~cm})$ en la medición del año 2016, midiendo su diámetro (forcípula Haglof Mantax) y altura total (HT; m) con el clinómetro Suunto PM-5/1520PC. El cálculo del área basal con la fórmula (Ecuador forestal, 2010).

$$
\mathrm{AB}=\frac{\pi \times \mathrm{D}^{2}}{4}
$$

Donde: $\mathrm{AB}=$ área basal; $\mathrm{D}=\mathrm{DAP}[$ Diámetro a la altura del pecho $(1.30$ $\mathrm{m})]$,

$\boldsymbol{\pi}=3.1416$ (constante)

La ecuación utilizada de volumen total del árbol en pie (Ecuador forestal, 2010).

$$
V=\frac{3.1416 \times(D A P)^{2} \times h \times f f}{4}
$$

Donde: $\mathrm{V}=$ volumen total del árbol $\left(\mathrm{m}^{3}\right)$, DAP = Diámetro del árbol a la altura del pecho $(\mathrm{m}) ; \mathrm{h}=$ Altura comercial o total del árbol (m), ff = factor de forma $=0.7$

La densidad de cobertura (D) mediante la ecuación(Alvarado, 2013).

$$
\mathrm{D}=\frac{\mathrm{AB}}{\mathrm{HT}} \times 100
$$

Donde: $\mathrm{D}=$ Densidad de cobertura en $\%, \mathrm{AB}=$ Área basal $\left(\mathrm{m}^{2} \mathrm{ha}^{-1}\right)$, $\mathrm{HT}=$ Altura total promedio de los árboles del rodal (m).

En el análisis de correlación entre el diámetro a la altura del pecho, la altura en función de la edad se aplicó la ecuación de la recta: $\mathrm{Y}=\mathrm{a}+\mathrm{bx}$ en donde la variable dependiente $\mathrm{Y}$ es el DAP, la altura total y la variable independiente $\mathrm{X}$ corresponde a la edad.

Para el cálculo del índice de raleo (IR) primeramente se utilizó la fórmula de varianza para datos agrupados

$$
\mathbf{S}^{2}=\frac{\sum \mathbf{X i}^{2} \mathbf{f i}-\left(\sum \mathbf{X i ~ f i}\right)^{2} / n}{n-1}
$$

Adicionalmente se calculó el límite inferior de confianza a un nivel de probabilidad estadística del valor tabular al 99,9\% para seleccionar los individuos que están bajo este valor y se determina el índice de raleo (IR) mediante la ecuación según (Vizcaino \& Aguirre, 2010, p.18).

$$
I R=\frac{\text { Número de individuos a extraerse }}{\text { Número total de individuos muestreados }} \times 100
$$




\section{Análisis estadístico}

Mediante el cálculo de estimadores estadísticos al procesar las variables dasométricas, el cálculo del índice de raleo. Se utilizó para los análisis la hoja de cálculo de Excel y el software Infostat (Balzarini et al., 2008).

\section{Resultados}

\section{Variables dasométricas}

El inventario demuestra que la masa arbórea está formada por una sola especie en etapa de desarrollo fustal con un DAP > a $8 \mathrm{~cm}$ se contabilizaron 1853,33 árboles ha ${ }^{-1}$; estos tienen un DAP promedio de 15,27 cm y una altura total promedio de $12,76 \mathrm{~m}$.

Tabla 1. Variables dasométricas con la estimación estadística.

\begin{tabular}{|c|c|c|c|c|c|c|c|}
\hline Parcelas & DAP & HTP & $\mathrm{ABP}$ & $\mathrm{N}$ & $\mathrm{AB} \mathrm{ha}^{-1}$ & $\mathrm{D}$ & VTPárbol $^{-1}$ \\
\hline & $\mathrm{cm}$ & $\mathrm{m}$ & $\mathrm{m}^{2}$ & & $\mathrm{~m}^{2}$ & $\%$ & \\
\hline $\mathrm{P} 1$ & 14,74 & 12,51 & 0,017 & 1880,00 & 32,09 & 256,54 & 0,15 \\
\hline $\mathrm{P} 2$ & 15,79 & 12,86 & 0,020 & 1760,00 & 34,47 & 267,93 & 0,18 \\
\hline $\mathrm{P} 3$ & 15,27 & 12,91 & 0,018 & 1920,00 & 35,16 & 272,32 & 0,17 \\
\hline Media & 15,27 & 12,76 & 0,02 & 1853,33 & 33,91 & 265,59 & 0,16 \\
\hline Dato ha-1 & & & & 1853,33 & 33,91 & 265,59 & 303,56 \\
\hline Desv. Est. & 0,52 & 0,22 & 0,00 & 83,27 & 1,61 & 8,14 & 0,01 \\
\hline Coef. Varia. & 3,43 & 1,72 & 6,85 & 4,49 & 4,74 & 3,07 & 8,24 \\
\hline Error. Muest. & 6,30 & 3,16 & 12,59 & 8,25 & 8,71 & 5,63 & 15,14 \\
\hline
\end{tabular}

Diámetro a la altura del pecho (DAP), Altura total promedio (HTP), Área basal promedio árbol ${ }^{-1}(\mathrm{ABP})$, Número de árboles fustales $(\mathrm{N})$, Área basal $h^{-1}\left(\mathrm{AB} \mathrm{ha}{ }^{-1}\right)$, Densidad de cobertura (D), Volumen total promedio árbol ${ }^{-1}$ $\left(\mathrm{VTPárbol}^{-1}\right)$.

Como se muestra en la tabla1, la media aritmética del área basal del bosque plantado para el $P$. radiata es de $0,02 \mathrm{~m}^{2}$ árbol ${ }^{-1}$, con 1.853,33 árboles, se estima $33,91 \mathrm{~m}^{2} \mathrm{ha}^{-1}$ de área basal y una densidad de cobertura del $265,59 \%$ de área basal con un volumen total de 303,56 $\mathrm{m}^{3} \mathrm{ha}^{-1}$.

\section{Relaciones de las variables DAP y altura total promedio en función de la edad}

El DAP del árbol presentó una alta correlación en $P$. radiata con una $\mathrm{r}$ de 0.99 , lo que demuestra un alto grado de asociación entre las variables diámetro a la altura del pecho y la edad (Tabla 2). 
Tabla 2. Modelo de regresión lineal de la variable DAP en función de la edad

\begin{tabular}{|rrrrrrrc|}
\hline Variable & $\mathrm{N}$ & $\mathrm{r}$ & $\mathrm{R}^{2}$ & $\mathrm{R}^{2}$ Ajus & $\mathrm{p}$ & Modelo \\
\hline $\mathrm{DAP}(\mathrm{cm})$ & 8 & 0,99 & 0,98 & 0,98 & 0,0001 & $\mathrm{Y}=2,03 \mathrm{X}-0,02$ \\
\hline
\end{tabular}

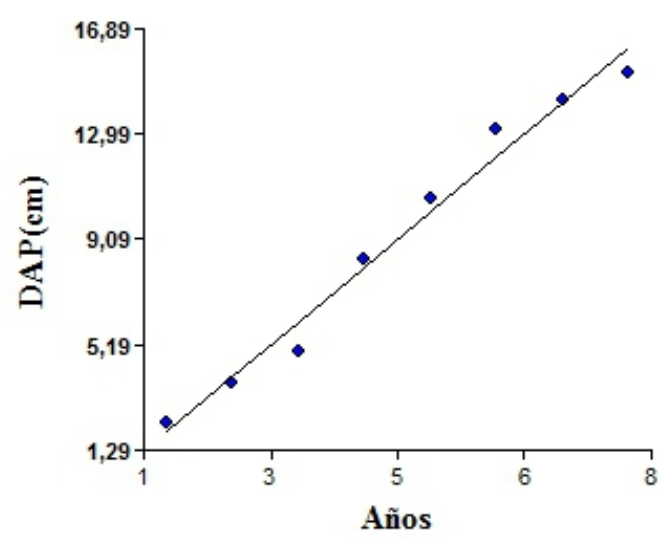

Figura 1. Diámetro a la altura del pecho-edad de la especie $P$. radiata

El DAP muestra una tendencia con desplazamiento hacia arriba conforme transcurre la edad, tal tendencia facilita la toma de decisiones en forma confiable sobre la aplicación de raleo en ésta especie (Figura 1).

En la Tabla 3 se indica que la altura total del árbol mostró una alta correlación en $P$. radiata con una $\mathrm{r}$ de 0.99 , lo que demuestra un alto grado de asociación entre las variables altura total promedio y la edad (Rodríguez, 2010).

Tabla 3. Modelo de regresión lineal de la variable altura total promedio por árbol en función de la edad

\begin{tabular}{|ccrcccc|}
\hline Variable & $\mathrm{N}$ & $\mathrm{r}$ & $\mathrm{R}^{2}$ & $\mathrm{R}^{2}$ Ajus & $\mathrm{p}$ & Modelo \\
\hline $\mathrm{HTP}(\mathrm{m})$ & 8 & 0,99 & 0,98 & 0,98 & 0,0001 & $\mathrm{Y}=1,82 \mathrm{X}-1,08$ \\
\hline
\end{tabular}

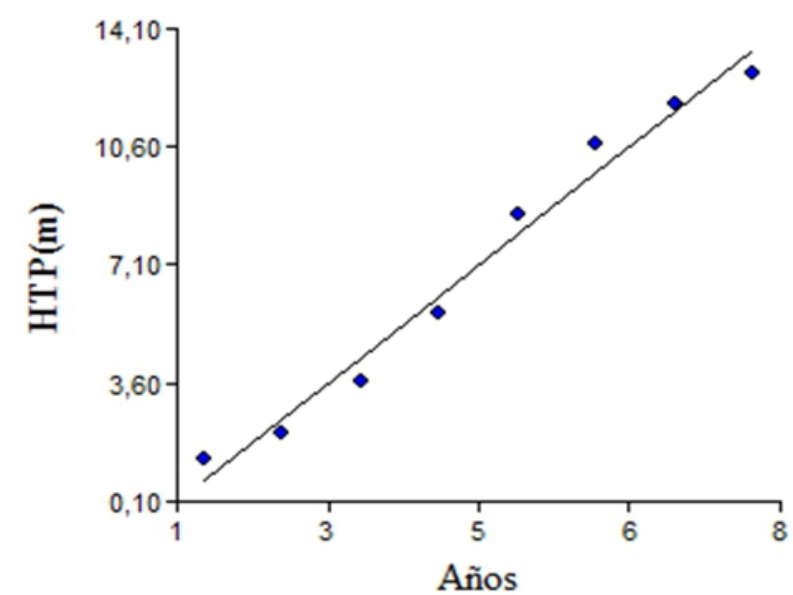

Figura 2. Curva altura total promedio-edad de $P$. radiata 
La altura total promedio muestra una tendencia con desplazamiento hacia arriba conforme transcurre la edad, tal tendencia facilita la toma de decisiones en forma confiable sobre la aplicación de la poda a los árboles de $P$. radiata en la plantación en estudio (Figura 2).

\section{Índice de raleo}

El índice de raleo reportó un valor de $28.77 \%$, calculado en base al límite inferior de confianza y el número de árboles que forman parte de las dos primeras clases diamétricas, cifra considerada técnicamente aceptable (Tabla 4).

Tabla 4. Índice de raleo en base al análisis de varianza de datos agrupados del DAP

\begin{tabular}{|c|c|c|c|c|c|}
\hline Clases & $\operatorname{Pmi}(\mathrm{Xi})$ & $\mathrm{Fi}$ & $\mathrm{Xi}^{2}$ & xifi & $\mathrm{Xi}^{2} \mathrm{fi}$ \\
\hline $8,34-11,34$ & 10,09 & 19 & 101,85 & 191,75 & 1027,88 \\
\hline $11,34-14,34$ & 12,80 & 38 & 163,87 & 486,44 & 2097,68 \\
\hline $14,34-17,34$ & 15,75 & 41 & 248,19 & 645,91 & 3909,97 \\
\hline $17,34-20,34$ & 18,50 & 28 & 342,32 & 518,05 & 6333,43 \\
\hline $20,34-23,34$ & 21,16 & 12 & 447,73 & 253,92 & 9473,83 \\
\hline \multirow[t]{2}{*}{$23,34-26,34$} & 24,61 & 1 & 605,42 & 24,61 & 14896,66 \\
\hline & 17,15 & 139 & 1909,38 & 2120,68 & 37739,46 \\
\hline Media & & & & $15,26 \mathrm{~cm}$ & \\
\hline Desv. Est. & & & & $6,25 \mathrm{~cm}$ & \\
\hline Coef. Varia. & & & & $40,94 \%$ & \\
\hline Error estándar & & & & $0,53 \mathrm{~cm}$ & \\
\hline Error. Est. Muest & & & & $1,78 \mathrm{~cm}$ & \\
\hline Límite inferior & & & & $13,48 \mathrm{~cm}$ & \\
\hline Índice de Raleo & & & & $28,77 \%$ & \\
\hline
\end{tabular}

Promedio del DAP de los árboles en cada clase (Pmi; Xi), Frecuencia absoluta (Fi).

\section{Discusión}

\section{Variables dasométricas}

El incremento medio anual estimado en DAP de $P$. radiata fue 1,22 veces superior al valor promedio de $1,57 \mathrm{~cm}$ año ${ }^{-1}$ reportado en Biobío-Chile con una precipitación media anual de $1.150 \mathrm{~mm}$ a una altitud $320 \mathrm{msnm}$, con pendiente del terreno de 36,5\% y 23 años de edad (Hubert et al., 2010) y demuestra un comportamiento similar al dato de $2,04 \mathrm{~cm}_{\text {año }}{ }^{-1}$ obtenido en un estudio en Mulaló a 3150 msnm (Paguanquiza, 2012).

La variable altura total promedio árbol ${ }^{-1}$ de la plantación en estudio resulto 0,45 veces menor en relación a los $28 \mathrm{~m}$ en un rodal de 23 años de edad (Hubert et al., 2010). El incremento medio anual estimado es similar en referencia al valor de $1,56 \mathrm{~m}_{\text {año }}{ }^{-1}$ a los 9 años de edad en Galicia (Sánchez \& Rodríguez, 2003). Asimismo coincide con el 1,53 m año ${ }^{-1}$, incremento 
determinado en un rodal de la misma especie sometido a raleos y podas en la parroquia Mulaló (Paguanquiza, 2012). En este sentido, uno de los factores que influyen en el incremento en altura total promedio y DAP, es la alta densidad que presenta la plantación, la altura es una de las variables que está influenciada por la densidad, lo que concuerda con lo que menciona (Klepac, 1983), quién sostiene que existe una tendencia a la reducción del incremento en altura en las plantaciones excesivamente densas.

El área basal promedio ha $^{-1}$ proyectado, reportó un valor de $33,91 \mathrm{~m}^{2}$ $\mathrm{ha}^{-1}$, ligeramente superior frente a $32,9 \mathrm{~m}^{2} \mathrm{ha}^{-1}$ en la región de Biobío-Chile (Hubert et al., 2010). A nivel de Ecuador en Mulaló es 1,61 veces mayor a los $21,15 \mathrm{~m}^{2} \mathrm{ha}^{-1}$ con una densidad de 980 árboles. Valor influenciado por la densidad de árboles, la cual produce un efecto directo sobre la productividad del sitio. Al aumentar la densidad se incrementa igualmente la producción, hasta un cierto límite (antes de la competencia intraespecífica). Por ello es importante considerar el raleo, como una redistribución de recursos en un número aceptable de individuos seleccionados bajo un cierto criterio técnico (Acosta, 2008).

La densidad de cobertura en función del área basal, presentó un valor de 265,59 \% superando al $90 \%$, indicando aplicar raleo inmediato a la plantación (Alvarado, 2013).

El volumen total promedio árbol ${ }^{-1} \mathrm{del}$ área de investigación es 12,56 veces el valor de 2,01 $\mathrm{m}^{3}$ árbol $^{-1}$ de un rodal de 23 años (Hubert et al., 2010). Comparativamente es 1,23 veces mayor al dato de $0,13 \mathrm{~m}^{3}$ árbol$^{-1}$ determinado en un bosque plantado en Mulaló (Paguanquiza, 2012). Asimismo su incremento medio anual estimado $\mathrm{ha}^{-1}$ año ${ }^{-1}$ resulto 1,59 veces mayor a los 23,7 $\mathrm{m}^{3} \mathrm{ha}^{-1}$ año $^{-1}$ en el País Vasco en suelos de textura franca (Bravo \& Montero, 2008).

\section{Relaciones de las variables DAP y altura total en función de la edad}

El método de correlación utilizado demostró un alto grado de asociación con un $\mathrm{r}$ de 0,99 en ambos casos entre las variables: DAP, altura total promedio frente a la variable independiente en este caso la edad cuyo modelo corresponde a una regresión lineal altamente significativa (Rodríguez \& Ramírez, 2016).

\section{Índice de raleo}

Este rodal en estudio por no haber tenido un primer raleo inicial de sanidad, los resultados silviculturales pueden ser desalentadores a corto y mediano plazo (Nalvarte, 2004). Por cuanto las copas de los árboles y sus ramas se interceptan, lo cual es un indicador que los individuos han iniciado su competencia intraespecífica. En este sentido el índice de raleo cercano al $30 \%$, es una cifra técnicamente aceptable y que amerita una extracción de 533 
árboles ha ${ }^{-1}$ seleccionando aquellos que han tenido un crecimiento no deseado por escaso manejo o la misma genética del individuo con la intención de homogenizar el rodal en términos de cantidad y calidad de árboles hectárea ${ }^{-1}$. Con el propósito de disminuir la presencia de limitaciones en luz, agua y nutrientes (Vizcaino \& Aguirre, 2010, p.18). A fin de concentrar el crecimiento y productividad en los mejores individuos (Meza \& Torres, 2006).

\section{Conclusiones}

La relación entre las variables DAP-edad y altura total promedio-edad fue altamente significativa, con coeficientes de correlación igual a 0,99 en la especie $P$. radiata. La ecuación de regresión que mejor estimó el crecimiento en diámetro y altura en función a la edad es la ecuación de tipo lineal.

La plantación en estudio tiene una alta densidad de árboles $\mathrm{ha}^{-1}$, área basal, volumen total, densidad en cobertura $>265,59 \%$, parámetros que sugieren ejecutar la actividad de raleo a una intensidad del $28,77 \%$ a fin de evitar su pérdida por competencia. En los árboles restantes luego del raleo se deberá aplicar el tratamiento silvicultural de poda.

\section{Agradecimiento}

A la empresa Haro Maderas, S.A por facilitar el bosque plantado y la información básica del mismo para la ejecución de la presente investigación.

\section{References:}

1. Acosta, C. (2008). Evaluación de una fertilización en Eucalyptus globulus Labill aplicada en la etapa de máxima acumulación nutritiva. (Trabajo de pregrado). Escuela de ingeniería Forestal. Valdivia, Chile: Universidad Austral de Chile.

2. Alvarado, M. (2013). Guía de silvicultura: Análisis y prescripción de comportamientos en planes de manejo con fines de silvicultura $y$ manejo forestal. Obtenido de http://icf.gob.hn/wpcontent/uploads/2015/08/GUIA-DE-SILVICULTURA.pdf

3. Balzarini, M. G., Gonzalez, L., Tablada, M., Casanoves, F., Di Rienzo, J. A., \& Robledo, C. W. (2008). Infostat. Manual del Usuario. Córdova, Argentina: Brujas.

4. Bravo, A., \& Montero, G. (2008). Descripción de los caracteres culturales de las principales especies forestales de España. Madrid, España: Instituto Nacional de Investigación y Tecnología Agraria y Alimentaria.INIA.

5. Carle, J., J, B., \& A, d. (2009). The Global Thematic Study of Planted Forests.In: Planted Forests.Uses,Impacts And Sustainability.J.Evans.FAO, CABI. 
6. Diaz Balteiro, L., Bertomeu, M., \& Giménez, J. (2008). Gestión óptima de Eucalyptus globulus en Galicia. Researchgate, 17.

7. Ecuador forestal. (2010). Normas para el manejo sostenible de los bosques húmedos. Obtenido de http://ecuadorforestal.org/wpcontent/uploads/2010/05/Normas-para-el-Manejo-Forestal-

Sostenible-de-los-Bosques-H\%C3\%BAmedos.pdf

8. Ecuador Forestal. (Julio de 2013). Organización Ecuador Forestal. Recuperado el 15 de Marzo de 2014, de http://ecuadorforestal,org/fichas-tecnicas-de-especiesforestales/ficha-tecnica-no-15-eucalyptus-globulus-labill/

9. Estación Meteorológica de la ESPOCH. (2014). Datos promedios de las variables:precipitación, temperatura y humedad relativa.

10. FAO. (2011). Diseños de muestreo de las Evaluaciones Forestales Nacionales.

Obtenido

de http://www.fao.org/fileadmin/user_upload/national_forest_assessmen t/images/PDFs/Spanish/KR2_ES_4_.pdf

11. Grijalva et al. (2012). Situación de los recursos genéticos forestales en Ecuador. Informe país Ecuador. Quito.

12. Hernández, J. (2014). Curso Regional de Regulación, Manejo y Salud Forestal. Chihuahua.

13. Hubert, A., Iroumé, A., Mohr, C., \& Frene, C. (2010). Efecto de plantaciones de Pinus radiata y Eucalyptus globulus sobre el recurso agua en la Cordillera de la Costa de la región del Biobío, Chile. Bosque, 31(3), 219-230.

14. Klepac, D. (1983). Crecimiento e incremento de árboles y masas forestales. Chapingo, México: Universidad Autónoma de Chapingo.

15. Laboratorio de suelos de la Facultad de Recursos Naturales de la ESPOCH. (2012). Resultados de análisis de suelos de la localidad Tahuala. Riobamba, Ecuador.

16. MAE.Ministerio de Agricultura, Ganadería, Acuacultura y Pesca. (2010). Aprovechamiento de los Recursos Forestales 2007-2009. Quito, Ecuador.

17. MAE. (2015). Programa de Incentivos para la Reforestación con Fines Comerciales. Guayaquil, Ecuador.

18. Meza, A., \& Torres, G. (2006). El raleo: una operación silvicultural fundamental, Costa Rica. Kurú: Revista Forestal, 3(8).

19. Nájera, J. A., \& Hernández, H. E. (2008). Relaciones morfométricas de un bosque coetáneo de la región del Salto, Durango. Ra Ximhai, 4 (1), 69-81.

20. Nalvarte, W. (2004). Silvicultura en la Amazonía Peruana. Diagnóstico de experiencias en la región de Ucayali y la provincia de Puerto Inca. CIFOR, IRENA, INIA, UN. Puculpalla, Perú. 
21. Paguanquiza, E. (2012). Elaboración de una línea base para determinar el crecimiento y desarrollo de las plantaciones de Pinus patula y Pinus radiata en la hacienda San Joaquin de Aglomerados Cotopaxi S.A (ACOSA)(Trabajo de pregrado). Escuela Superior Politécnica de Chimborazo, Chimborazo, Ecuador.

22. Palacios, W., \& Quiroz, H. (2012). Sondeo de percepciones sobre la rentabilidad del aprovechamiento de madera por pequeños propietarios. Proyecto USAID Costas y Bosques sostenibles. Quito, Ecuador.

23. Rodríguez, H., \& Ramírez, H. (2016). Dendrometria (forest mensuration) Medición forestal. ResearchGate.

24. Rodríguez, M. (2010). Correlación y Regresión. Diseño de Experimentos y Modelos de Regresión Lineal: Aplicaciones en SPSS. Maule, Colombia: Universidad Católica de Maule. Facultad de Ciencias Básicas.

25. Sánchez, F., \& Rodríguez, R. (2003). Selvicultura de Pinus radiata. Compostela: Universidad de Santiago de Compostela.

26. Sierra, R. (1999). Propuesta preliminar de un sistema de clasificación para el Ecuador Continental. Quito, Ecuador: Proyecto INEFAN/GEF-BIRF y EcoCiencia.

27. Sharma, M., Amateis, R., \& Burkhart, H. (2001). Top height definition and its effect on site index determination in thinned and unthinned loblolly pine plantations in: Forest Ecology and Management.

28. Vizcaino, M., \& Aguirre, C. (2010). “Aplicación de Estimadores Estadísticos y Diseños Experimentales en Investigaciones Forestales. Ibarra, Ecuador. 